\title{
A NOTE ON ESTIMATING DISTRIBUTION FUNCTIONS ${ }^{1}$
}

\author{
J. R. BLUM
}

1. Statement of the problem. Let $A$ be a positive number and for each positive integer $n$ let $f_{n}(y)$ be a continuous function on the closed interval $[-A, A]$. Let $F(y)$ be a distribution function on $[-A, A]$. For each $n=1,2, \cdots$, define $a_{n}$ by

$$
a_{n}=\int_{-A}^{A} f_{n}(y) d F(y) .
$$

In this note we consider the problem of estimating the distribution function $F(y)$ in terms of the sequence of numbers $\left\{a_{n}\right\}$, and the sequence of functions $f_{n}(y)$. To this end we consider, for each positive integer $n$, a system of equations and inequalities. We construct a distribution function $F_{n}(y)$ in terms of any solution of this system, and show that $\lim _{n \rightarrow \infty} F_{n}(y)=F(y)$ for every continuity point of $F(y)$.

2. Conditions for uniqueness of $F$. It is clear that in order to be able to estimate $F$, we must assume that $F$ is the unique distribution function satisfying (1.1). More precisely we shall make the following

Assumption. Let $G(y)$ be any function of bounded variation defined on $[-A, A]$ and satisfying

$$
a_{n}=\int_{-A}^{A} f_{n}(y) d G(y), \quad n=1,2, \cdots
$$

Then $F(y)-G(y)$ is identically constant.

In this section we shall derive a condition which is equivalent to the uniqueness assumption. To this end let $B$ be the Banach space of continuous functions defined on $[-A, A]$ and normed by

$$
\|f\|=\max _{y \in[-A, A]}|f(y)| \text {. }
$$

Then we have

THEOREM 1. A necessary and sufficient condition that $F$ be unique is that the sequence $f_{n}(y)$ be fundamental in $B$.

Proof. Suppose that $F$ is unique. Let $B^{\prime}$ be the closed linear manifold spanned by the sequence $f_{n}$, and suppose that $B^{\prime}$ is a proper

Received by the editors February 28, 1955.

${ }^{1}$ Sponsored by the Office of Ordnance Research, U. S. Army, under Contract No. DA-33-008-ORD-965. 
subspace of $B$. Let $f_{0} \in B-B^{\prime}$, and let $\phi$ be a bounded linear functional defined on $B$ with $\phi\left(f_{0}\right)=1$, and $\phi(f)=0$, for $f \in B^{\prime}$. It is well known that such functionals exist. From the representation theorem for linear functionals on $B$ it follows that there exists a function of bounded variation on $[-A, A]$, say $H(y)$, satisfying

$$
\phi(f)=\int_{-A}^{A} f(y) d H(y), \quad \text { for every } f \in B .
$$

Now let $G(y)=F(y)+H(y)$. Clearly $H(y)$ is not identically constant, for $\int_{-A}^{A} f_{0}(y) d H(y)=1$. On the other hand we have

$$
a_{n}=\int_{-A}^{A} f_{n}(y) d G\left(y^{\prime}\right), \quad n=1,2, \cdots,
$$

since $\int_{-A}^{A} f_{n}(y) d H(y)=0$ for every $n$. Since $F$ is assumed to be unique, it follows that the sequence $f_{n}$ is fundamental, thus proving necessity.

Conversely suppose that the sequence $F_{n}$ is fundamental in $B$, and suppose that $G(y)$ is a function of bounded variation on $[-A, A]$ satisfying

$$
\int_{-A}^{A} f_{n}(y) d F(y)=\int_{-A}^{A} f_{n}(y) d G(y), \quad n=1,2, \cdots .
$$

From the fact that strong convergence in $B$ implies weak convergence in $B$, and from the fact that the sequence $f_{n}$ is fundamental in $B$, it follows that equation (2.5) holds for every $f \in B$. Hence for every real number $t$ we have

$$
\int_{-A}^{A} e^{i t y} d F(y)=\int_{-A}^{A} e^{i t y} d G(y),
$$

and the uniqueness of $F$ follows from well-known properties of Fourier-Stieltjes transforms.

3. Construction of the sequence $F_{n}$. Let $n$ be a given positive integer. Let $y_{0}=-A, y_{1}, y_{2}, \cdots, y_{n}=A$ be a subdivision of $[-A, A]$ into $n$ equal subintervals. For $1 \leqq i \leqq n, 1 \leqq j \leqq n$, define the numbers $M_{i j}^{(n)}$ and $m_{i j}^{(n)}$ by

$$
M_{i j}^{(n)}=\max _{\nu_{j-1} \leqq y \leqq \nu_{j}} f_{i}(y), \quad m_{i j}^{(n)}=\min _{\nu_{j-1} \leqq \nu \leqq \nu_{j}} f_{i}(y) .
$$

Consider the following system of equations and inequalities in the unknowns $H_{1}^{(n)}, \cdots, H_{n}^{(n)}$ : 

(i) $H_{j}^{(n)} \geqq 0$,
$j=1, \cdots, n$.
(ii) $\sum_{j=1}^{n} H_{j}^{(n)}=1$.
$\begin{array}{rlrl}\text { (iii) } & \sum_{j=1}^{n} M_{i j}^{(n)} H_{i}^{(n)} \geqq a_{i}, & i=1, \cdots, n . \\ \text { (iv) } \sum_{j=1}^{n} m_{i j}^{(n)} H_{j}^{(n)} \leqq a_{i}, & i=1, \cdots, n .\end{array}$

$(3.2)$

The system (3.2) clearly has the solution $H_{j}^{(n)}=\int_{y_{j-1}}^{y_{i}} d F, j=1, \cdots, n$. Now let $H_{1}^{(n)}, \cdots, H_{n}^{(n)}$ be an arbitrary solution of (3.2). We define a distribution function $F_{n}(y)$ on $[-A, A]$ by

$$
F_{n}(y)=\sum_{y j \leqq y} H_{j}^{(n)} .
$$

In the next section we shall prove

Theorem 2. For each point of continuity of $F(y)$ we have

$$
\lim _{n \rightarrow \infty} F_{n}(y)=F(y) \text {. }
$$

\section{Proof of Theorem 2.}

LEMMA 1. Let $r$ be a fixed positive integer. Then $\lim _{n \rightarrow \infty} \int_{-A}^{A} f_{r}(y) d F_{n}(y)$ $=a_{r}$.

PROOF. We have $\int_{-A}^{A} f_{r}(y) d F_{n}(y)=\sum_{j=1}^{n} f_{r}\left(y_{j}\right) H_{j}^{(n)}$ for every positive integer $n$. Hence

$$
\sum_{j=1}^{n} m_{r j}^{(n)} H_{j}^{(n)} \leqq \int_{-A}^{A} f_{r}(y) d F_{n}(y) \leqq \sum_{j=1}^{n} M_{r j}^{(n)} H_{j}^{(n)},
$$

and it is sufficient to show that

$$
a_{r}=\lim _{n \rightarrow \infty} \sum_{j=1}^{n} m_{r j}^{(n)} H_{j}^{(n)}=\lim _{n \rightarrow \infty} \sum_{j=1}^{n} M_{r j}^{(n)} H_{j}^{(n)} .
$$

Now for each $n \geqq r$, we have, in virtue of (3.2),

Also

$$
\sum_{j=1}^{n} m_{r j}^{(n)} H_{j}^{(n)} \leqq a_{r} \leqq \sum_{j=1}^{n} M_{r j}^{(n)} H_{j}^{(n)} .
$$

$$
\sum_{j=1}^{n}\left[M_{r j}^{(n)}-m_{r j}^{(n)}\right] H_{j}^{(n)} \leqq \max _{\jmath=1, \ldots, n}\left[M_{r j}^{(n)}-m_{r j}^{(n)}\right]
$$


Since $f_{r}(y)$ is uniformly continuous on $[-A, A]$, the desired result follows.

Lemma 2. For each $f \in B$, we have

$$
\lim _{n \rightarrow \infty} \int_{-A}^{A} f(y) d F_{n}(y)=\int_{-A}^{A} f(y) d F(y) .
$$

Proof. Let $f \in B$, and let $\epsilon$ be a positive number. The sequence $f_{n}$ is fundamental in $B$, and so we may choose a finite subset, say $f_{i_{1}}, \cdots$, $f_{i_{r}}$, and real numbers $c_{1}, \cdots, c_{r}$ with the property that

$$
\left\|f-\sum_{j=1}^{r} c_{j} f_{i j}\right\|<\epsilon / 3 \text {. }
$$

Without loss of generality we may assume that $\sum_{i=1}^{r}\left|c_{j}\right|>0$. Now, from Lemma 1 , we may choose an integer $N$, so that for $n \geqq N$ we have

$$
\max _{j=1, \ldots, n}\left|\int_{-A}^{A} f_{i j}(y) d F_{n}(y)-\int_{-A}^{A} f_{i_{j}}(y) d F(y)\right|<\epsilon / 3 \sum_{j=1}^{r}\left|c_{j}\right|
$$

and consequently

$$
\mid \int_{-A}^{A}\left(\sum_{j=1}^{r} c_{j} f_{i_{j}}(y)\right) d F_{n}(y)-\int_{-A}^{A}\left(\sum_{j=1}^{r} c_{j} f_{i_{j}}(y)\right) d F(y)<\frac{\epsilon}{3} .
$$

Then for $n \geqq N$, we have

$$
\begin{aligned}
&\left|\int_{-A}^{A} f(y) d F_{n}(y)-\int_{-A}^{A} f(y) d F(y)\right| \\
& \leqq\left|\int_{-A}^{A} f(y) d F_{n}(y)-\int_{-A}^{A}\left(\sum_{j=1}^{r} c_{j} f_{i_{j}}(y)\right) d F_{n}(y)\right| \\
&+\left|\int_{-A}^{A}\left(\sum_{j=1}^{r} c_{j} f_{i_{j}}(y)\right) d F_{n}(y)-\int_{-A}^{A}\left(\sum_{j=1}^{r} c_{j} f_{i_{j}}(y)\right) d F(y)\right| \\
&+\left|\int_{-A}^{A}\left(\sum_{j=1}^{r} c_{j} f_{i_{j}}(y)\right) d F(y)-\int_{-A}^{A} f(y) d F(y)\right| .
\end{aligned}
$$

The first and last terms are bounded by $\left\|f-\sum_{j=1}^{r} c_{j} f_{i j}\right\|$, and the middle term by $\epsilon / 3$. Hence

$$
\left|\int_{-A}^{A} f(y) d F_{n}(y)-\int_{-A}^{A} f(y) d F(y)\right|=\epsilon .
$$


The proof of the theorem is now immediate. For each real number $t$, let $\psi_{n}(t)=\int_{-A}^{A} e^{i t y} d F_{n}(y)$, and let $\psi(t)=\int_{-A}^{A} e^{i t y} d F(y)$. Then we have $\lim _{n \rightarrow \infty} \psi_{n}(t)=\psi(t)$ for every $t$, and the theorem follows from the continuity theorem for Fourier-Stieltjes transforms.

I wish to thank Professor E. W. Barankin of the University of California for several helpful discussions concerning this problem.

INDIANA UNIVERSITY

\section{REAL-VALUED MAPPINGS OF SPHERES}

E. E. FLOYD

This note concerns subsets $\Delta$ of the unit 2-sphere $S$ such that $\left({ }^{*}\right)$ for each continuous real-valued mapping $f$ of $S$ there exists a rotation $r$ of $S$ with all points of $r(\Delta)$ having the same value under $f$. In 1942, Kakutani [3] proved that the set $\Delta$ of end points of an orthonormal set of 3 vectors has property $\left({ }^{*}\right)$. It was observed by de Mira Fernandes [5] that the same proof holds in case $\Delta$ is the set of vertices of any equilateral triangle. Yamabe and Yujobo [8] proved a generalization of Kakutani's theorem to $n$-space. Their method may be used to prove that the set $\Delta$ of vertices of an isosceles triangle has property $\left({ }^{*}\right)$ (this has been carried out in a Master's thesis of R. D. Johnson [2]). Here we prove that the set $\Delta$ of vertices of any triangle has property $\left({ }^{*}\right)$; the methods differ from both those of Kakutani and those of Yamabe and Yujobo.

Dyson [1] has proved that the set of vertices of a square centered at the origin has property $\left({ }^{*}\right)$; Livesay [4] has extended this to any rectangle centered at the origin. The problem of finding all such sets $\Delta$ having property $\left({ }^{*}\right)$ is unsolved.

THEOREM. Let $f$ be a continuous real-valued mapping of the sphere $S$ and let $x_{0}, x_{1}, x_{2} \in S$. There exists a rotation $r$ with $f\left(r\left(x_{0}\right)\right)=f\left(r\left(x_{1}\right)\right)$ $=f\left(r\left(x_{2}\right)\right)$.

We need the following lemma.

Lemma. Suppose that $X$ is a unicoherent locally connected continuum, and that $T$ is a map of period 2 on $X$ without fixed points. Suppose $A$ is a subset of $X$ which (i) is closed in $X$, (ii) is invariant under $T$, and

Received by the editors September 23, 1954. 